\title{
Estudio comparativo entre catéteres peritoneales autoposicionantes y catéteres Tenckhoff en referencia a su desplazamiento intraperitoneal
}

\author{
$M^{\text {a }}$ Carmen Salvador Lengua, $M^{\mathrm{a}}$ José Folch Morro, Alexandra Mallol Domínguez, Jorge \\ Bort Castelló, Elena Renau Ortells, Vicente Cerrillo García, Carmen Aicart Saura
}

Enfermeros/as. Unidad de Diálisis. Hospital General de Castellón

\section{Resumen}

El avance en el ámbito de los accesos en la diálisis peritoneal plantea cuestiones que requieren su discernimiento y su posible solución. Una de las causas más comunes de los problemas mecánicos es el atrapamiento por el omento o el estreñimiento. Otros factores que pueden afectar al buen funcionamiento de un catéter de diálisis peritoneal es la existencia de adherencias y/o hernias en la zona abdominal.

En nuestra unidad de diálisis peritoneal se utilizan catéteres autoposicionantes y no autoposicionantes (Tenckhoff), por lo que decidimos realizar un estudio a fin de comparar que diferencias existen en su vida de funcionamiento.

Nuestro objetivo ha sido comparar las diferencias existentes referidas a complicaciones por desplazamiento y malposición entre los dos tipos de catéteres. Y valorar si la obesidad, la cirugía abdominal previa y el tiempo de permanencia son variables que influyen en el buen funcionamiento de los catéteres.

La muestra estudiada pertenece a pacientes que actualmente están en activo en el programa de diálisis peritoneal en nuestra unidad.

Correspondencia:

$\mathrm{M}^{\mathrm{a}}$ Jose Folch Morro

$\mathrm{C} /$. Maestro Goterriz N ${ }^{\circ} 7,4^{\circ}$

12540, Villarreal, Castellón

e-mail: mjfolki@gmail.com
Se contabilizaron 35 pacientes. El $60 \%$ era portador de catéter autoposicionante y el $40 \%$ Tenckhoff. Un $37.4 \%$ de los pacientes había sido intervenido a nivel abdominal y/o pélvico. Solo un $28.57 \%$ eran obesos (con un Índice de Masa Corporal> 30) con una media de peso de $72.8 \pm 18.9 \mathrm{Kg}$.

Los catéteres fueron implantados con cirugía abierta, anestesia local y sedación por lo que se realizaba de forma ambulatoria.

Para la elección del catéter se utilizó una correlación numérica aleatoria.

El catéter Tenckhoff es un tubo de silicona con múltiples orificios distales y que puede terminar de forma recta o enroscada. También presenta un dacron para evitar las infecciones del orificio.

El catéter autoposicionante tiene la misma forma que un catéter Tecnckoff pero con una modificación. En su extremo distal tiene un aumento del diámetro de unos dos centímetros, que es el añadido de un peso de 12 gramos de un material biocompatible (tungsteno) que favorece que el catéter esté siempre en la parte inferior de la cavidad peritoneal, evitando el mal funcionamiento por desplazamiento.

Para analizar las complicaciones mecánicas de ambos tipos de catéteres recogimos las ocasiones en las que se utilizaba un evacuante intestinal (X-Prep) y en las que había sido necesaria una recolocación en quirófano (utilizando únicamente un fiador para su recolocación y sin necesidad de anestesia local).

Se realizó una hoja de recogida de datos en la que se anotaron todas las variables: sexo, edad, tiempo en 
diálisis peritoneal, índice de masa corporal, número de desplazamientos, intervenciones abdominales previas, y según los resultados obtenidos se confirma estadísticamente que los catéteres autoposicionantes presentan menos problemas mecánicos de migración del catéter, que los catéteres Tenckhoff.

Aunque se puede apreciar que en pacientes obesos existe una mayor tolerancia a ambos tipos de catéter apareciendo un menor número de complicaciones.

También se observa que ambos catéteres tienen más problemas a partir del primer año de su implantación, siendo significativo a partir de los 24 meses de permanencia. Esto nos confirma la buena colocación de los catéteres por parte del cirujano, ya que al inicio de su implantación hay menos complicaciones.

Los problemas mecánicos que han presentado ambos tipos de catéteres se han solucionado administrando X-Prep o mediante la recolocación. Sólo hubo un paciente que se tuvo que recurrir a cirugía abierta para cambiar el catéter siendo este del tipo autoposicionante. Observando que el problema fue ocasionado por las múltiples adherencias que presentaba sin haber sido intervenido anteriormente en la zona abdominal.

Este caso nos ha llevado a plantear que los pacientes pueden presentar problemas por atrapamiento, no solo por las intervenciones quirúrgicas, sino también por su fisiología.

En este estudio no se ha comparado el dolor en los dos tipos de catéteres. Se podría realizar otro estudio valorando esta variable, ya que el peso existente en la parte distal del catéter autoposicionante podría aumentar la sensación de dolor en los pacientes.

\section{PALABRAS CLAVE:}

\section{- CATÉTER AUTOPOSICIONANTE}

- CATÉTER TENCKOFF

- COMPLICACIONES

- DESPLAZAMIENTO

\section{Comparative study between self-locating peri- toneal catheters and Tenckhoff catheters with regard to intraperitoneal displacement}

\section{Abstract}

The advances made in the field of accesses in peritoneal dialysis raise questions that require discernment and a possible solution. One of the most common causes of mechanical problems is omental entrapment or constipation. Other factors that can affect correct functioning of a peritoneal dialysis catheter is the existence of adhesions and/or hernias in the abdominal area.

In our peritoneal dialysis unit, self-locating and non-self-locating (Tenckhoff) catheters are used, and therefore we decided to carry out a study to compare the differences in their operating life.

Our aim was to compare the differences concerning complications due to displacement and incorrect positioning between the two types of catheter, and also to evaluate whether obesity, prior abdominal surgery and the time the catheter is in place are variable that affect correct functioning of the catheters.

The sample studied comprised patients who are currently active on the peritoneal dialysis programme in our unit.

A total of 35 patients were studied. $60 \%$ had a selflocating catheter and $40 \%$ a Tenckhoff catheter. $37.4 \%$ of the patients had undergone abdominal and/ or pelvic surgery. Only $28.57 \%$ were obese (with a Body Mass Index $>30$ ) with an average weight of $72.8 \pm 18.9 \mathrm{Kg}$.

The catheters were implanted using open surgery, local anaesthetic and sedation and therefore in on an ambulatory basis.

Random numerical correlation was used for the choice of catheter.

The Tenckhoff catheter is a silicon tube with multiple distal orifices and which can have a straight or threaded tip. It also has a Dacron cuff to prevent infections of the orifice. 
The self-locating catheter has the same form as a Tenckhoff catheter but with a modification. At the distal tip it has an increased diameter of two centimetres, which is the result of adding a 12-gramme weight made of a biocompatible material (tungsten) to favour the positioning of the catheter always in the lower part of the peritoneal cavity, avoiding malfunction due to displacement.

To analyse the mechanical complications of both types of catheters we recorded the occasions on which a bowel evacuant (X-Prep) was used and in which surgical repositioning of the catheter had been necessary (using only a guidewire to re-position it and without the need for local anaesthetic).

A data compilation sheet was drawn up on which all the variables were noted: sex, age, time on peritoneal dialysis, body mass index, number of displacements, prior abdominal operations, and according the results obtained statistical confirmation was obtained that self-locating catheters present fewer mechanical problems of catheter migration than Tenckhoff catheters.

Although it can be seen that in obese patients there is greater tolerance to both types of catheter, with fewer complications.

It was also observed that both catheters present more problems after one year following implantation, significantly after 24 months in place. This confirms to us the good placing of the catheters by the surgeon, as at the beginning of implantation there are fewer complications.

The mechanical problems presented by both types of catheter have been overcome by administering X-Prep or by re-positioning. There was only one patient who required open surgery to change the catheter, which was self-locating, and it was observed that the problem was caused by the multiple adhesions the patient present without having previously undergone surgery in the abdominal area.

This case has led us to consider that patients may present entrapment problems not just because of surgical operations, but also because of their physiology.
This study did not compare pain in the two types of catheter. Another study could be carried out evaluating this variable, as the weight in the distal tip of the self-locating catheter could increase the sensation of pain experienced by patients.

\section{KEY WORDS:}

- SELF-LOCATING CATHETER

- TENCKHOFF CATHETER

- COMPLICATIONS

- DISPLACEMENT

\section{Introducción}

El avance en el ámbito de los accesos en la diálisis peritoneal plantea cuestiones que requieren su discernimiento y su posible solución. Una de las causas más comunes de los problemas mecánicos es el atrapamiento por el omento o el estreñimiento (los fecalomas pueden desplazar el catéter) ${ }^{(1)}$. Con el fin de evitarlo, la porción del catéter intraperitoneal se dirige hacia el cuadrante inferior izquierdo, ya que, el peristaltismo del colon descendente reduce la migración del catéter. También una dieta rica en fibra va a facilitar la motilidad intestinal favoreciendo el buen posicionamiento del catéter. No hay que olvidar la correcta cocción de los alimentos para eliminar el socio ( $\mathrm{Na}$ ) y el potasio (K). Uno de los factores que afecta al buen funcionamiento de un catéter de diálisis peritoneal es la existencia de adherencias y/o hernias en la zona abdominal.

En nuestra unidad de diálisis peritoneal se utilizan catéteres autoposicionantes y no autoposicionantes (Tenckhoff), por lo que decidimos realizar un estudio, entre ambos tipos de catéteres, a fin de comparar que diferencias existen ante la complicación de desplazamientos que sufren en su vida de funcionamiento. Un buen posicionamiento favorece la permeabilidad del catéter siendo condicionante del éxito de diálisis peritoneal.

Existen estudios que afirman que el catéter autoposicionante presenta menor porcentaje de malposiciones respecto al catéter Tenckhoff ${ }^{(2,3,4)}$. 
Los catéteres a estudio eran portadores de un solo dacron.

\section{Objetivo}

Comparar las diferencias existentes referidas a complicaciones por desplazamiento y malposición entre los dos tipos de catéteres.

Valorar si la obesidad, la cirugía abdominal previa y el tiempo de permanencia son variables que influyen en el buen funcionamiento de los catéteres.

\section{Material y métodos}

La muestra estudiada pertenece a pacientes que actualmente están en activo en el programa de diálisis peritoneal en nuestra unidad.

Se contabilizaron 35 pacientes de los cuales 17 eran mujeres $(48.57 \%)$ y 18 hombres $(51.43 \%)$, con una media de edad de 50 años y 7 meses \pm 25 años.

El $60 \%$ de los pacientes eran portadores de catéter autoposicionante y el $40 \%$ Tenckhoff.

Un $37.4 \%$ de los pacientes había sido intervenido a nivel abdominal y/o pélvico.

Solo un $28.57 \%$ eran obesos (con un IMC > 30) con una media de peso de $72.8 \pm 18.9 \mathrm{Kg}$.

Los catéteres fueron implantados con cirugía abierta a través de la línea media infraumbilical, con anestesia local y sedación por lo que se realizaba de forma ambulatoria, no siendo necesario el ingreso.

Para la elección del catéter se utilizó una correlación numérica aleatoria y según la pauta siguiente: de arriba a bajo y de izquierda a derecha. Si el número que correspondía era par se colocaba un catéter autoposicionante y si era impar no autoposicionante (Tenckhoff).

El protocolo de nuestra unidad en un postimplante para comprobar su permeabilidad es:

- Comprobación en el quirófano durante el implante de su permeabilidad, infundiendo suero fisiológico
(SF) con una jeringa de 50cc, que realiza el cirujano. Seguidamente se le dice al paciente que tosa, para comprobar que drene el SF.

- En la habitación inmediatamente después de quirófano se realizan lavados de \pm 200 cc de líquido peritoneal a temperatura ambiente, dejando, si sale claro, 200cc más lcc de Heparina sódica al $1 \%$ intraperitoneal. Así se comprueba que no se ha lesionado ningún vaso sanguíneo ${ }^{(5)}$.

- Se repite el mismo procedimiento cada siete días hasta completar el mes.

El catéter Tenckhoff es un tubo de silicona con múltiples orificios distales y que puede terminar de forma recta 0 enroscada. También presenta un dacron para evitar las infecciones del orificio ${ }^{(5)}$.

El catéter autoposicionante tiene la misma forma que un catéter Tecnckoff pero con una modificación. En su extremo distal tiene un aumento del diámetro de unos dos centímetros, que es el añadido de un peso de 12 gramos de un material biocompatible (tungsteno) que favorece que el catéter esté siempre en la parte inferior de la cavidad peritoneal, evitando el mal funcionamiento por desplazamiento ${ }^{(6)}$.

Para analizar las complicaciones mecánicas de ambos tipos de catéteres recogimos las ocasiones en las que se utilizaba un evacuante intestinal (X-Prep) y en las que había sido necesaria una recolocación en quirófano (utilizando únicamente un fiador para su recolocación y sin necesidad de anestesia local).

Se realizó una hoja de recogida de datos en la que se anotaron todas las variables: sexo, edad, tiempo en diálisis peritoneal, IMC, número de desplazamientos, intervenciones abdominales previas.

\section{Análisis estadísticos}

El análisis estadístico se realizó con el programa G-Stat, utilizando para la comparación de las variables cuantitativas los test de kruskal Wallis y $\mathrm{U}$ Mann-Whitney, y para las variables cuantitativas el Chi cuadrado. 


\section{Resultados}

Según los resultados obtenidos se confirma estadísticamente que los catéteres autoposicionantes presentan menos problemas mecánicos de migración del catéter, que los catéteres Tenckhoff (tabla 1).

\begin{tabular}{|c|c|c|}
\hline & $\begin{array}{c}\text { NO AUTOPO- } \\
\text { SICIONANTE }\end{array}$ & $\begin{array}{c}\text { AUTOPOSI- } \\
\text { CIONANTE }\end{array}$ \\
\hline TIPO CATÉTER & 14 & 21 \\
\hline MEDIA & 1,93 & 0,57 \\
\hline P-valor de U: 0.0264 1. Siendo $p$ significativo $p<0.05$ \\
\hline
\end{tabular}

Tabla 1. Relación del tipo de catéter con la utilización de X-Prep

A nivel estadístico no existen diferencias significativas en relación con las variables de la obesidad y las intervenciones quirúrgicas previas a la implantación del catéter peritoneal (tabla 2 y 3 ).

\begin{tabular}{|c|c|c|}
\hline & NO & SÍ \\
\hline OBESOS & 25 & 10 \\
\hline MEDIA & 1,36 & 0,5 \\
\hline P-valor de U: 0.2705 . Siendo $p$ significativo $p<0.05$ & \\
\hline
\end{tabular}

Tabla 2. Relación de la obesidad con la utilización de X-Prep

\begin{tabular}{|c|c|c|}
\hline & NO & SÍ \\
\hline INTERVENIDOS & 22 & 13 \\
\hline MEDIA & 1,09 & 1,15 \\
\hline P-valor de U: 0.8338 . Siendo $p$ significativo $p<0.05$ & \\
\hline
\end{tabular}

Tabla 3. Relación de la cirugía previa con la utilización de X-Prep

Aunque se puede apreciar que en pacientes obesos existe una mayor tolerancia a ambos tipos de catéter apareciendo un menor número de complicaciones.

También se observa que ambos catéteres tienen más problemas a partir del primer año de su implantación, siendo significativo a partir de los 24 meses de permanencia (tabla 4).

\begin{tabular}{|c|c|c|c|c|}
\hline MESES & 0 a 1 & 1 a 6 & 6 a 12 & $>12$ \\
\hline MEDIA & 1,33 & 1,71 & 2 & 2,33 \\
\hline
\end{tabular}

\begin{tabular}{|c|c|c|}
\hline & $\begin{array}{c}>24 \text { MESES } \\
\text { PERMANENCIA }\end{array}$ & $\begin{array}{c}<24 \text { MESES } \\
\text { PERMANENCIA }\end{array}$ \\
\hline $\begin{array}{c}\text { UTILIZACION } \\
\text { X-PREP }\end{array}$ & $25.7 \%$ & $17,1 \%$ \\
\hline $\begin{array}{c}\text { NO UTILIZACION } \\
\text { X-PREP }\end{array}$ & $14,3 \%$ & $42,8 \%$ \\
\hline Chi-cuadrado 0.036 , siendo p significativo $p<0.05$ & \\
\hline
\end{tabular}

Tabla 4. Relación del tiempo de permanencia del catéter con la utilización de X-Prep.

\section{Discusión}

Los problemas mecánicos que han presentado ambos tipos de catéteres se han solucionado administrando X-Prep o mediante la recolocación. Sólo hubo un paciente que se tuvo que recurrir a cirugía abierta para cambiar el catéter siendo este del tipo autoposicionante. Observando que el problema fue ocasionado por las múltiples adherencias que presentaba sin haber sido intervenido anteriormente en la zona abdominal.

Este caso nos ha llevado a plantear que los pacientes pueden presentar problemas por atrapamiento, no solo por las intervenciones quirúrgicas, sino también por su fisiología.

En este estudio no se ha comparado el dolor en los dos tipos de catéteres. Se podría realizar otro estudio valorando esta variable, ya que el peso existente en la parte distal del catéter autoposicionante podría aumentar la sensación de dolor en los pacientes.

Hemos observado una mayor incidencia de complicaciones a partir del primer año de implantación del catéter. Esto nos confirma la buena colocación de los catéteres por parte del cirujano, ya que al inicio de su implantación hay menos complicaciones. 


\section{Conclusión}

En este estudio se demuestra que los catéteres autoposicionantes presentan menos problemas mecánicos.

La obesidad y las intervenciones quirúrgicas previas no son variables que puedan crear complicaciones a la hora del buen funcionamiento del catéter, ni en la necesidad de su recolocación. on-line en nuestros pacientes.

Recibido: 30 de Octubre 2011

Revisado: 30 Noviembre 2011

Modificado: 7 Agosto 2012

Aceptado 30 Agosto 2012

\section{Bibliografía}

1. M. García Martínez, $M^{a}$ Teresa Sola García, Raquel Pelayo Alonso. La posición del catéter de diálisis peritoneal no es esencial para un correcto funcionamiento. En: Libro de comunicaciones presentadas al XXXIII Congreso Nacional Seden. SEDEN 2008.

2. B. Millán Díaz, M. J. Castro vilanova, M. Hidalgo Díaz. Catéter autoposicionante. Experiencia clínica y estudio comparativo con el catéter tenckhoff. En: Libro de comunicaciones presentadas al XXXIII Congreso Nacional Seden. SEDEN 2008.

3. Cavagna $\mathrm{R}$; Tessarin C; Tarroni G. The self-locating catheter: clinical evaluation andcomparison with the Tenckhoff catheter. Perit Dial Int 1999 NovDec;19(6):540-3.

4. Di Paolo N; Capotondo L; Sansoni E; Romolini V. The self-locating catheter: clinical experience and follow-up. Perit Dial Int 2004 JulAug;24(4):359-64.

5. R. Rivas Martín, M.I. Sánchez Martín. Diálisis Peritoneal. En: Tratado de Enfermería en cuidados críticos pediátricos y neonatales (ECCPN).Capítulo 143 [acceso el 30 de Julio de 2012]. Disponible en: http://www.eccpn.aibarra.org/temario/ sección9.htm

6. J. L. Martín Espejo. Catéteres peritoneales. Tipos de catéteres Protocolos de implantación de catéteres peritoneales del grupo de D.P. de Andalucía. En: Diálisis Peritoneal. IV Curso andaluz de diálisis peritoneal para enfermería. Seden 2006. Cap. $5(32-41)$. 